\title{
An improved mouse orthotopic bladder cancer model exhibiting progression and treatment response characteristics of human recurrent bladder cancer
}

\author{
TOMOHARU NAITO ${ }^{1,2^{*}}$, TAMAMI HIGUCHI ${ }^{1,3^{*}}$, YASUHIRO SHIMADA $^{1,2}$ and CHIHAYA KAKINUMA ${ }^{1,2}$ \\ ${ }^{1}$ Pharmaceutical and Healthcare Research Laboratories, FUJIFILM Corporation, Ashigarakami-gun, Kanagawa 258-8577; \\ ${ }^{2}$ Department of Human Pathology, School of Medicine, Juntendo University, Tokyo 113-0033; ${ }^{3}$ Department of Oncology \\ Clinical Development, Gunma University, Graduate School of Medicine, Maebashi, Gunma 371-8511, Japan
}

Received April 23, 2019; Accepted September 10, 2019

DOI: $10.3892 / \mathrm{ol} .2019 .11172$

\begin{abstract}
Nonmuscle-invasive (superficial) bladder cancer is generally treated via surgical removal, followed by adjuvant therapy (bacillus Calmette-Guerin). However, bladder cancer can often recur, and in a substantial number of recurrent cases, the cancer progresses and metastasizes. Furthermore, residual microtumors following excision may lead to an increased risk of recurrence. An in vivo model mimicking the pattern of urinary bladder microtumor regrowth may provide an effective experimental system for improving postsurgical treatment outcomes. A mouse bladder cancer model established using orthotopic transplant of UM-UC-3 human urinary bladder carcinoma cells has been established, however, to the best of our knowledge, no report has investigated sequential histological changes, including early-phase changes and treatment responses in bladder cancer. In the present study, the efficiency of the model was optimized and the sequential changes were examined using histopathology and in situ imaging. The therapeutic effects of cisplatin (CDDP) and gemcitabine (GEM) were also examined, which are drugs that are often used for follow-up chemotherapy. Tumor-seeding efficiency reached
\end{abstract}

Correspondence to: Dr Tomoharu Naito, Pharmaceutical and Healthcare Research Laboratories, FUJIFILM Corporation, 577 Ushijima, Kaisei-machi, Ashigarakami-gun, Kanagawa 258-8577, Japan

E-mail: tomoharu.naito@fujifilm.com

*Contributed equally

Abbreviations: $\mathrm{BCG}$, bacillus Calmette-Guerin; CIS, carcinoma in situ; CDDP, cisplatin; EDTA, ethylenediaminetetraacetic acid; EMEM, Eagle's minimum essential medium; GEM, gemcitabine; $\mathrm{H} \& \mathrm{E}$, hematoxylin and eosin; TURBT, transurethral resection of bladder tumor

Key words: pathology, bladder cancer, superficial bladder cancer, orthotopic mouse model, microtumor, cisplatin, gemcitabine, chemotherapy
90-100\%, with muscle layer and bladder lumen invasion occurring in $\sim 21$ days, using the following modifications: i) Shallow catheter insertion to mitigate bladder wall damage; ii) bladder pretreatment using prewarmed trypsin, followed by light urethral clamping and body temperature maintenance for more efficient removal of transitional epithelium; and iii) seeding with UM-UC-3 cells (rather than HT1376, 5637 or T24 tumor cells) in a medium supplemented with Matrigel. Transplant with UM-UC-3 cells resulted in isolated microlesions that progressed into tumors, invading the bladder lumen and muscle layer to the serosal surface. Tumor growth was markedly reduced by weekly intravenous injections of CDDP and partially suppressed by GEM. Therefore, this model is reliable, and pathological progression and treatment responses recapitulate the features of recurrent human bladder cancer.

\section{Introduction}

Bladder cancer is derived from transitional bladder epithelium (1). Most bladder cancers ( $\sim 70 \%)$ are nonmuscle-invasive or superficial carcinomas and subclassified as either papillary urothelial neoplasm or carcinoma in situ (CIS) $(2,3)$. Endoscopic treatment with transurethral resection of bladder tumor (TURBT) in the early stage is the first-line strategy for diagnosis, staging, and treatment (4). However, TURBT is not effective for CIS because the disease is often diffuse and difficult to visualize. Thus, recurrence rate is high despite timely surgical removal, and recurrence may result in metastatic transition to muscle-invasive carcinoma (5-9).

At present, the therapeutic options for the recurrent cancer are intravesical administration of chemotherapeutic drugs, immunotherapy using bacillus Calmette-Guerin (BCG), and repeat urinary bladder resection (4). However, there is no consensus regarding drug selection, drug dose, or number of intravesical administrations (10). Mitomycin C is frequently adopted for intravesical treatment in the United States. In the European Union, many studies have been conducted on the anticancer efficacy of intravesical anthracycline (11).

An animal model of urinary bladder microtumor development would be invaluable for analyzing tumor-progression mechanisms and evaluating treatment efficacy. Orthotopic 
transplantation of human bladder cancer cells into the mouse bladder provides such a model; however, it remains unclear whether this model adequately mimics the pathological progression and treatment responses of human bladder cancer. Indeed, evaluation of an orthotopic mouse bladder cancer model implanted with the human urothelial carcinoma cell line UM-UC-3 has been limited to luminescence and end-point assays, such as histopathology. However, sequential histological examination for early-phase changes in and progression of bladder cancer have not been reported (12-15).

In the present study, we evaluated histopathological changes from microtumor and superficial carcinoma to invasive carcinoma in the mouse urinary bladder following orthotopic transplantation of human bladder cancer cells. Additionally, we examined the therapeutic efficacy of the widely used antitumor drugs cisplatin (CDDP) and gemcitabine (GEM). Reportedly, tumor engraftment and pathology strongly depend on the transplantation protocol (12-20). Accordingly, we improved the protocols for urinary bladder pretreatment, catheterization, and other experimental conditions.

\section{Materials and methods}

Cell cultures and animals. Human bladder cancer cell lines UM-UC-3, T24, HT1376, and 5637 were provided by American Type Culture Collection. UM-UC-3 and HT1376 lines were cultured in Eagle's minimum essential medium (EMEM; Wako), 5637 cells in RPMI1640 (Gibco, Thermo Fisher Scientific, Inc.), and T24 cells in McCoy's 5A medium (Gibco, Thermo Fisher Scientific, Inc.). In all the cultures, the medium was supplemented with streptomycin $(100 \mathrm{mg} / \mathrm{ml})$ and penicillin (100 units/ml; Pen strep; Gibco, Thermo Fisher Scientific) as well as with $10 \%$ fetal bovine serum (FBS; Gibco, Thermo Fisher Scientific, Tokyo, Japan). Cells were maintained in a humidified incubator with $5 \% \mathrm{CO}_{2}$ at $37^{\circ} \mathrm{C}$.

We purchased 7-week-old female C.B-17/IcrHsd-Prkdcscid mice from Japan SLC (Hamamatsu, Japan). These animals were transferred to a temperature-controlled $\left(20-26^{\circ} \mathrm{C}\right)$ and humidity-controlled (40-60\%) room with a 12-h light/12-h dark cycle during the experimental period. All animal experiments were approved by the FUJIFILM Animal Experimentation Committee.

Orthotopic implantation of human bladder cancer cell lines. Cultured human bladder cancer cells were carefully harvested from culture plates using a scraper (Sumitomo Bakelite Co., Ltd.) without trypsin/EDTA and washed once with FBS-supplemented medium and twice with serum-free medium. Cells were then suspended at $1 \times 10^{7}$ cells $/ 100 \mu 1$ in serum-free EMEM and Matrigel (1:1; Corning Incorporated Life Sciences) on ice prior to orthotopic transplantation.

The orthotopic animal model was established using a previously described technique $(21,22)$ with several modifications (described below). Briefly, female mice were anesthetized with $1-2 \%$ isoflurane (Pfizer). Anesthetized mice were placed at $37^{\circ} \mathrm{C}$ on a hot plate in the supine position for all transplant procedures. To prevent infection, the urethral tip was cleaned with $70 \%$ ethanol (Wako), and a 24-gauge Terumo catheter (Terumo) was inserted through the urethra into the bladder. The bladder was washed three times with $100 \mu \mathrm{l}$ phosphate-buffered saline (PBS;
Gibco, Thermo Fisher Scientific, Inc.). To prevent scratches inside the bladder, the catheter tip was inserted only up to $1 \mathrm{~cm}$ from the urethral meatus (Fig. 1A). Then, two boluses of $100 \mu \mathrm{l}$ of $0.25 \%$ trypsin EDTA (Gibco; Thermo Fisher Scientific, Inc.) at $37^{\circ} \mathrm{C}$ were infused into the bladder under anesthesia, with each infusion retained for $30 \mathrm{~min}$ using a 20 -g pressure clip (KN-353 AS-1, Natsume Seisakusho Co., Ltd.) on the urethra. The trypsin solution was then drained and the bladder washed twice with $100 \mu 1$ of EMEM. Finally, $100 \mu \mathrm{l}$ EMEM:Matrigel (1:1) containing $1 \times 10^{7}$ bladder cancer cells was infused into the bladder and retained for $2 \mathrm{~h}$ using the $20 \mathrm{-g}$ pressure clip under anesthesia. The clip was then removed and the urethral meatus treated with povidone-iodine for disinfection.

To evaluate the effects of trypsin treatment, we isolated the bladders from three untreated and three trypsin-treated mice and compared histological sections of their bladder wall. We then orthotopically transplanted the UM-UC-3 cell line ( $1 \times 10^{7}$ cells), as described, to evaluate the time course of tumor growth. In total, we utilized 40 mice to establish the tumor model in the bladder. UM-UC-3 (1×10 ${ }^{7}$ cells) model mice and pathologically examined tumor growth in each bladder 1 , $3,4,8,14,21$, and 28 days after the transplant. To evaluate bladder growth, we measured excised organ weight $3,5,14$, and 28 days after the transplant.

In our animal model, the maximum tumor diameter was approximately $1 \mathrm{~cm}$. In other cases, many tumors with a diameter of a few millimeters were diffusely distributed in the urinary bladder. The sum of the tumor burden was less than $1 \mathrm{~cm}$ in diameter. The mice exhibited lethal pathology comprising ureteral obstruction, followed by hydronephrosis due to severe kidney disease after continuous breeding for 28 days. Our institute's ethical code recommends that animals with moribundity (marked reduction in body weight, hypothermia, significant temperature drop, significant exhaustion (crouching position) must be euthanized immediately. The animals with moribundity were euthanized by blood-letting under isoflurane anesthesia (induction: $2.0-3.0 \%$ maintenance: $0.5-1.5 \%$ ) without any other pain (23). Death was confirmed by observation of respiratory and cardiac arrest. Therefore, our animal study complied with the code of ethical conduct approved by the FUJIFILM Animal Experimentation Committee.

CT. A 200- $\mu 1$ bolus of 5-fold diluted Iopamilon (Bayer) was intravenously administered under anesthesia and the urethral meatus closed with a surgical clip (20 g) for $20 \mathrm{~min}$. Computed tomography (CT) images were acquired using a three-dimensional (3D) micro-CT system (RmCT; Rigaku Co., Tokyo, Japan) with acquisition settings of $90 \mathrm{~V}$ and $100 \mu \mathrm{A}$ and $17 \mathrm{~s}$ of exposure. Tomographic images were obtained using i-VIEW 3D imaging software (Morita Co.) (24).

Evaluation of CDDP and GEM antitumor efficacy. CDDP was obtained from Sigma-Aldrich and GEM from Teva Pharmaceutical Industries. In this efficacy study, 32 mice was used, of which 24 mice were orthotopically inoculated with $1 \times 10^{7}$ UM-UC-3 cells (day 0) and then randomly divided into three groups:, a vehicle group receiving weekly injections of PBS, a CDDP group receiving weekly intravenous injection of $10 \mathrm{mg} / \mathrm{kg}$ CDDP at 7 and 14 days after cell transplant, and a GEM group receiving weekly intravenous injections of 

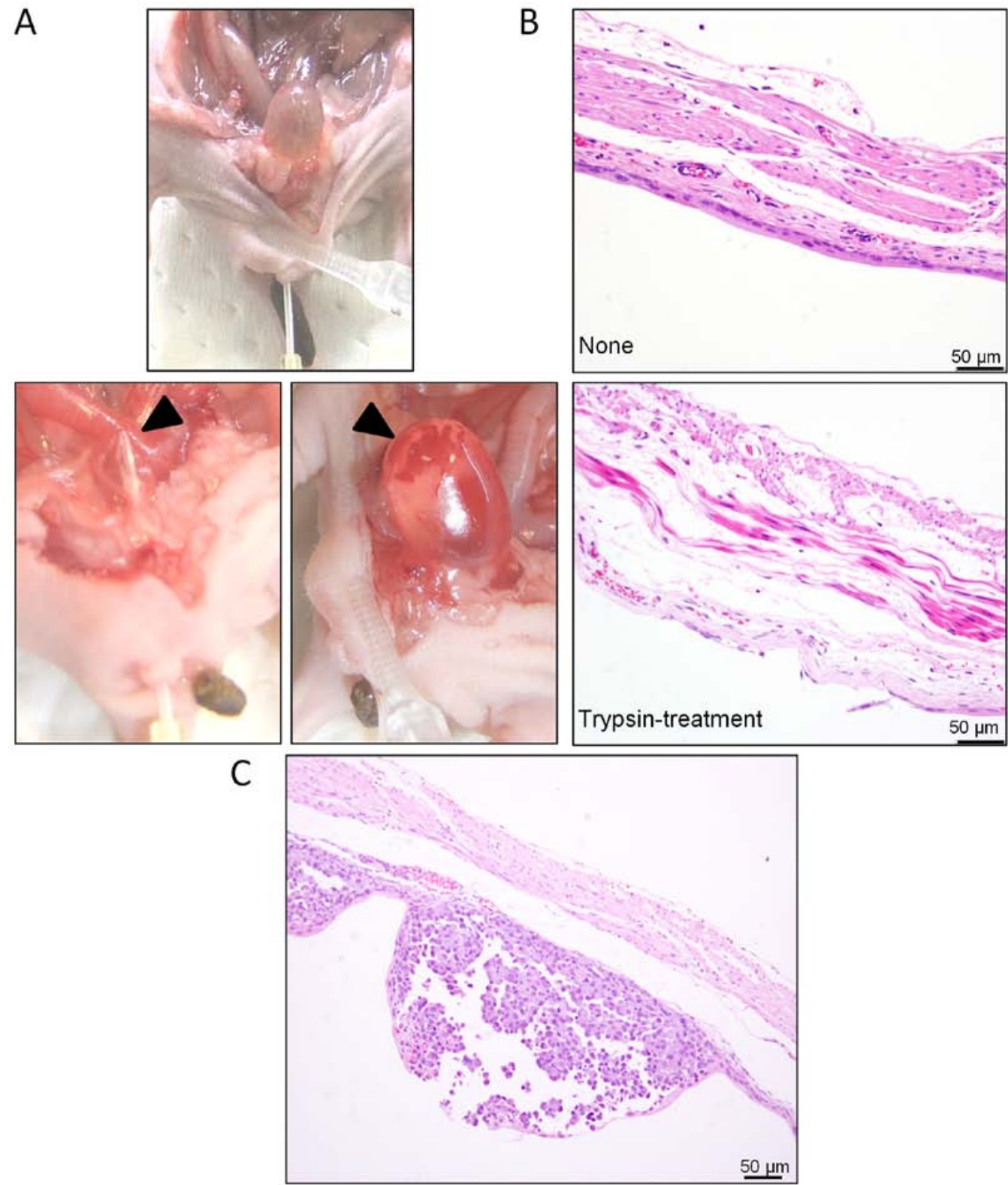

Figure 1. Technical refinement of the mouse orthotopic bladder cancer model. (A) Images presenting the typical depth of catheter insertion into the urinary bladder. Overinsertion can cause bladder hemorrhage. (B) Histological section of the bladder wall showing that modified trypsin treatment efficiently removed $>50 \%$ of the transitional epithelium (magnification, $\mathrm{x} 200$ ). (C) Histological image presenting the tumor nodules on the surface of the bladder wall after the transplantation of bladder cancer cells (magnification, $\mathrm{x} 200$ ).

$240 \mathrm{mg} / \mathrm{kg}$ GEM on days 7 and 14 after the transplant. The non-treatment group (normal) comprised eight mice that did not receive any transplants and injections. Reportedly, in mice, 10 and $240 \mathrm{mg} / \mathrm{kg}$ are the maximum tolerable doses of CDDP and GEM, respectively, for weekly treatment $(25,26)$. We euthanized and dissected mice from each group under anesthesia on day 21 after transplantation to examine treatment responses. Each group comprised eight animals for analysis due to intervening death and severe morbidity.

Bladder weight measurement and hematoxylin and eosin staining. After excision, the bladder was fixed by injecting $10 \%$ neutral buffered formalin (Wako), clipping the opening, and dipping the entire organ in formalin. After fixation, the bladder was opened up along the median, the formalin washed way, and the tissue weighed. Tissue samples were cut into 4-10 longitudinal strips (depending on the bladder size) approximately $2 \mathrm{~mm}$ in width. Specimens were embedded in paraffin (Sakura Finetek Japan) and 2- $\mu \mathrm{m}$ sections were prepared. The sections were stained with hematoxylin and eosin (H\&E; Hematoxylin 3G, Sakura Finetek Japan; Eosin, Wako) using standard procedures (hematoxylin for $1 \mathrm{~min}$, followed by eosin for $1 \mathrm{~min}$ ). Photomicrographs were obtained using an Olympus BX51 microscope equipped with an Olympus DP70 camera and cellSens software.

Statistical analysis. Group means were compared using one-way analysis of variance Dunnett's multiple comparison test. All statistical calculations were performed using GraphPad Prism 5.04 software (GraphPad Software, Inc.). $\mathrm{P}<0.05$ (two-tailed) was considered to indicare a significant difference. 
Day 1

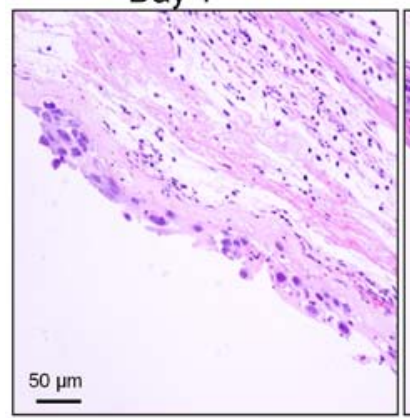

Day 8

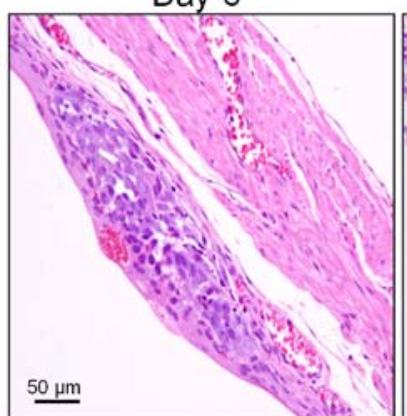

Day 21 (low)

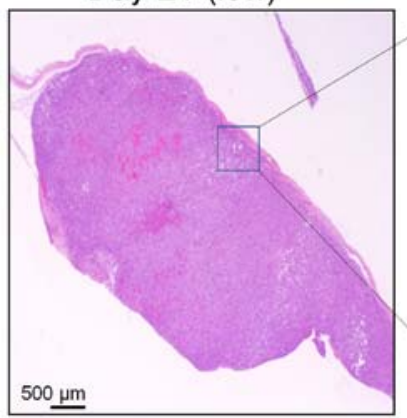

Day 3

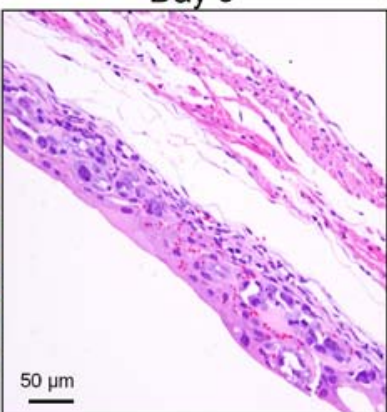

Day 14

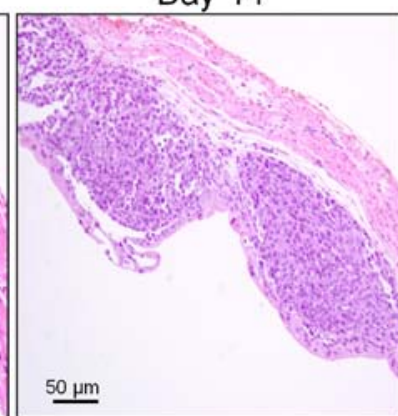

Day 21 (high)

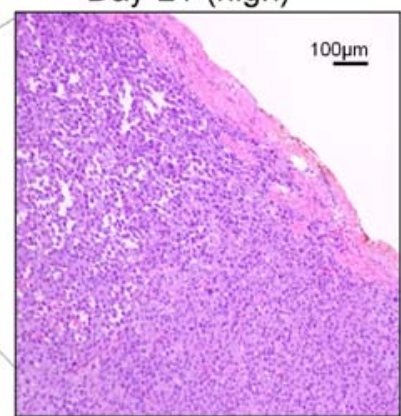

Figure 2. Sequential changes in bladder histology following orthotopic transplant of human bladder cancer cells. Images acquired $1,3,4$, and 8 days after transplant (magnification, x200), and images acquired 14, 21 (low power), and 21 days (high power) after transplant (magnification, x200, x40 and x100).

\section{Results}

Technical refinement of the mouse orthotopic bladder cancer model. To better reflect the pathological features and progression of human superficial bladder cancer in mice, several steps involved in orthotopic transplantation were modified, including catheter insertion depth, urethral ligation method, and intravesical trypsin reaction temperature. The reagents for trypsin pretreatment of the urinary bladder were prewarmed to $37^{\circ} \mathrm{C}$, and the treatment was performed on a hot plate to stably maintain body temperature and thus trypsin activity.

After pretreatment, the urethra was gently ligated with a surgical clip (20 g) so as not to induce necrosis, as observed in the urethral meatus following strong compression (data not shown). In such cases, the bladder dilated, hydronephrosis was induced, and the mouse became moribund. Furthermore, catheter insertion depth was controlled, with the tip limited to approximately $1 \mathrm{~cm}$ from the urethral meatus to prevent injury to the bladder wall (Fig. 1A, upper panel). If touched by the catheter tip, the wall was easily damaged and bled (Fig. 1A, lower panel). However, in our transplantation experiments using shallow catheter insertion, urinary bladder epithelium remained completely free from bleeding (Fig. 1B).
Furthermore, implantation of tumor cells following these modified steps reliably resulted in superficial bladder cancer or CIS (Fig. 1C).

In addition, multiple cell lines were examined for the most efficient tumor induction. The HT1376, 5637, T24, and UM-UC-3 cell lines were implanted in different groups, and tumor formation was histologically examined. Implantation of T24 tumor cells did not result in tumor formation, and only 50\% of mice implanted with 5637 or HT1376 tumor cells showed tumor foci in bladder. Moreover, the growth rate was too low to visibly observe the tumor foci 21 days after implantation. Under microscopy, the tumor showed partial cancer pearls and keratinocytes in squamous cell carcinoma. On the other hand, up to $90 \%$ of infused UM-UC-3 cells formed tumor nodules on the bladder wall (data not shown).

Sequential evaluation of cancer growth through histopathological examination, tissue weight measurement, and CT imaging. Under optimized conditions, $>50 \%$ of the trypsinized transitional epithelium was removed, and lamina propria of the mucous membrane appeared slightly edematous (Fig. 1B). Implanted cancer cells with condensed, atypical nuclei and eosinophilic cytoplasm formed tumor foci on the mucosal 


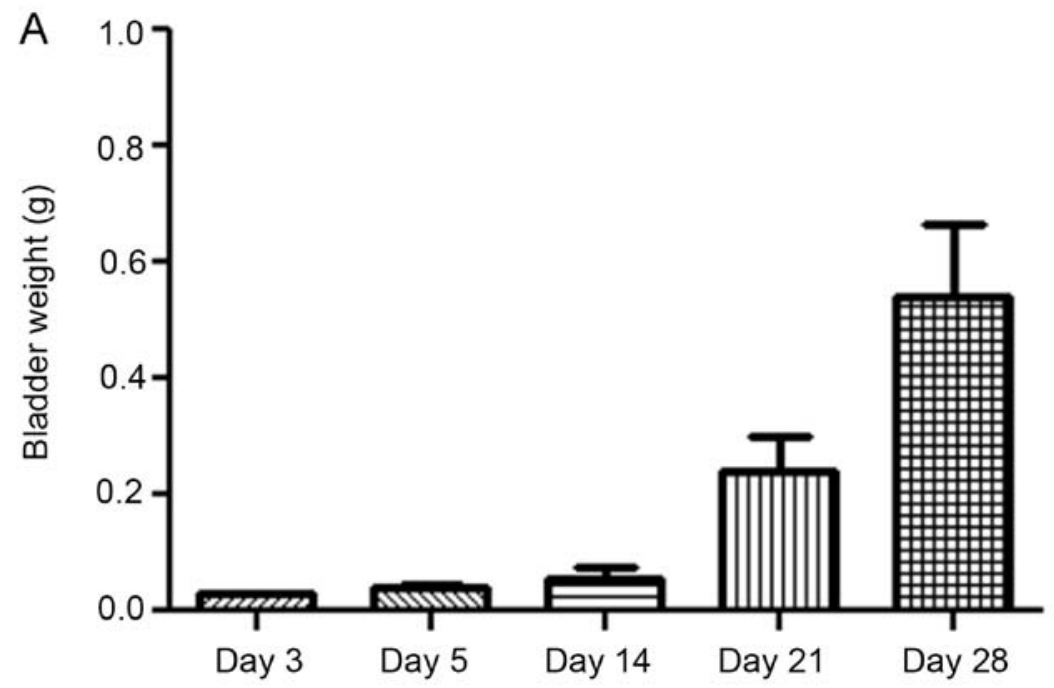

$\mathrm{B}$

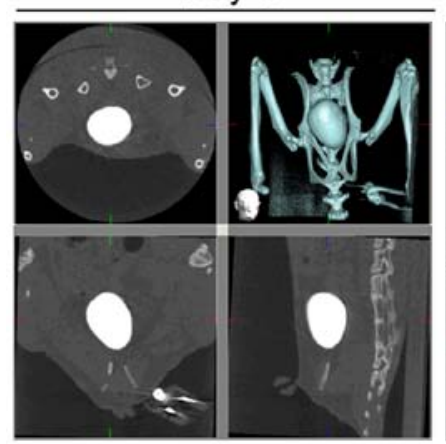

Day 14

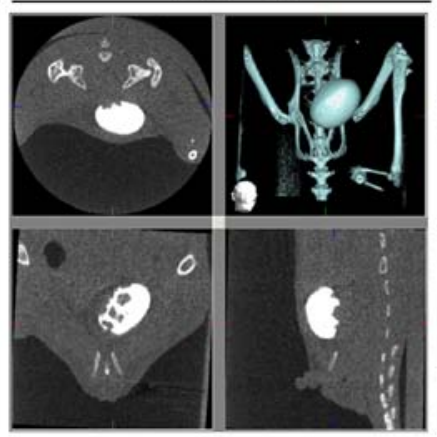

Day 21

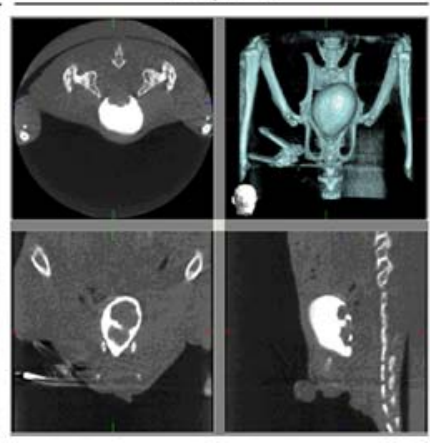

Figure 3. Bladder weight analysis and CT scanning to estimate tumor size. (A) Bladder weight 3, 5, 14, 21 and 28 days after transplantation. (B) CT images at 0,14 and 21 days after transplantation.

lamina propria 1 day after implantation. After 3 days, tumor foci were completely covered with transitional epithelium. Tumor foci rapidly increased in size and number from days 4 to 8 after transplantation, and individual tumor cells showed multiple cell divisions, but no invasive cancer cells were observed at this time.

By day 14 after transplantation, however, the tumor nodule had invaded the submucosal layer of the urinary bladder. On day 21 after transplantation, the tumor nodule appeared larger and mucinous and was accompanied by necrotic cells and bleeding. Furthermore, the tumor had invaded the bladder lumen and muscularis, even reaching the serosal surface (Fig. 2). Bladder weight rapidly increased over the first 14 days after transplantation (Fig. 3). CT scan revealed cancer nests protruding from the dorsal surface of the bladder into the bladder lumen on the 14th and 21st days after transplantation.

Histopathological analysis of anticancer drug treatment. To further validate this modified mouse orthotopic bladder cancer model, we assessed the effects of intravenous application of CDDP and GEM. Drug efficacy was evaluated on the basis of bladder weight and pathological findings. In the vehicle group, tumors were well developed by day 21 after transplantation, featuring sporadic cell necrosis, bleeding, and increased mucous production. Invasion was visible in both the bladder lumen and muscular layer, reaching the serosal surface. In the CDDP treatment group, there were fewer and smaller tumor lesions localized in the bladder wall; single-cell necrosis was more diffuse and the density of cancer cells was significantly reduced compared with that in the vehicle group. In the GEM treatment group, single-cell necrosis and reduction of cancer-cell density were more modest, and in most cases, proliferative and solid-tumor foci were still observed (Fig. 4).

Growth in the organ weight was observed in the vehicle group, whereas it was significantly suppressed in the CDDP treatment group. In fact, bladder weight was reduced almost to the level of normal transplant-naïve mice. In the GEM treatment group, the progressive bladder weight increase following transplantation was suppressed by approximately $50 \%$ compared with that in the vehicle group (Fig. 5). One mouse in the vehicle group was found dead due to the increase in tumor volume in the bladder.

\section{Discussion}

This is the first report of a mouse orthotopic bladder cancer model replicating the core features of human bladder cancer recurrence following incomplete excision. Implantation of UM-UC-3 human bladder cancer cells produced microtumors 


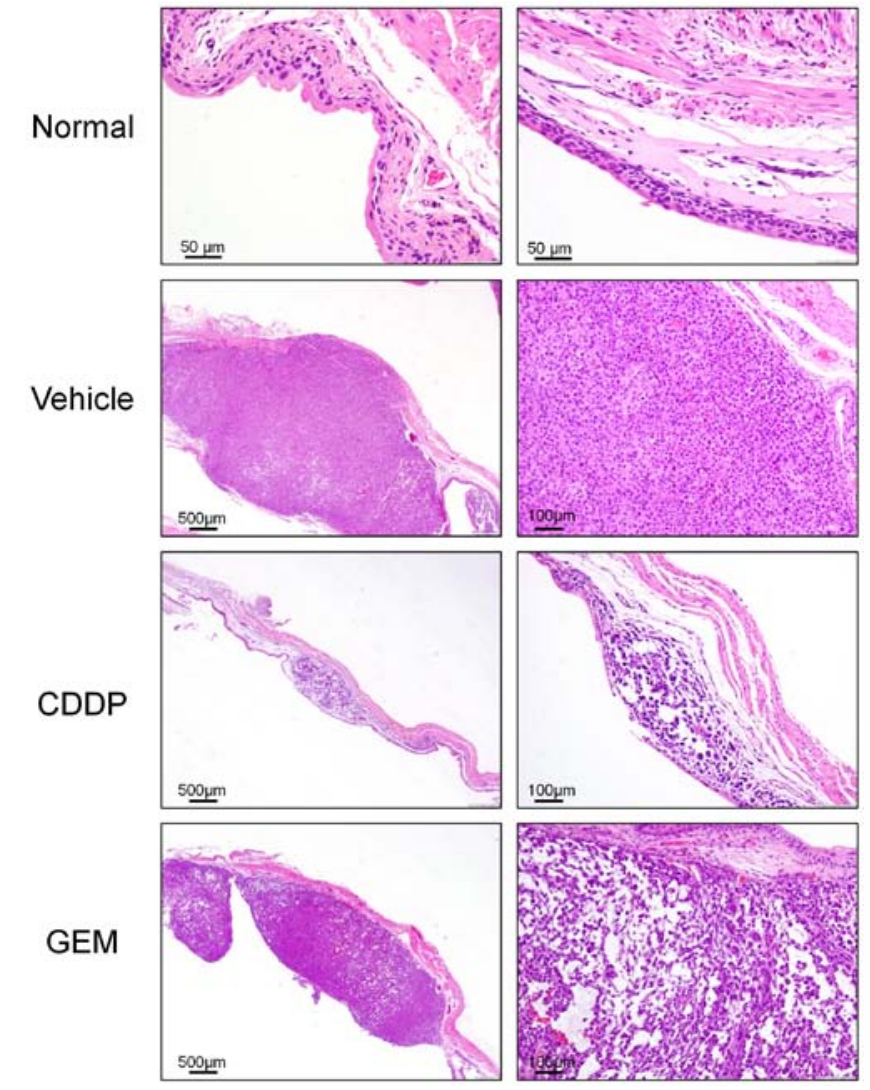

Figure 4. Microscopic bladder tumors in model mice after chemotherapy. Images of bladder tumor tissues following chemotherapy. Normal, nontransplanted mouse (magnification, x200); Vehicle, Vehicle(PBS)-treated mouse (magnification, $\mathrm{x} 40, \mathrm{x} 100$ ); CDDP, CDDP-treated mouse (magnification, $\mathrm{x} 40 \mathrm{x}, \mathrm{x} 100$ ); GEM, GEM-treated mouse (magnification, $\mathrm{x} 40, \mathrm{x} 100)$. CDDP, cisplatin; GEM, gemcitabine.

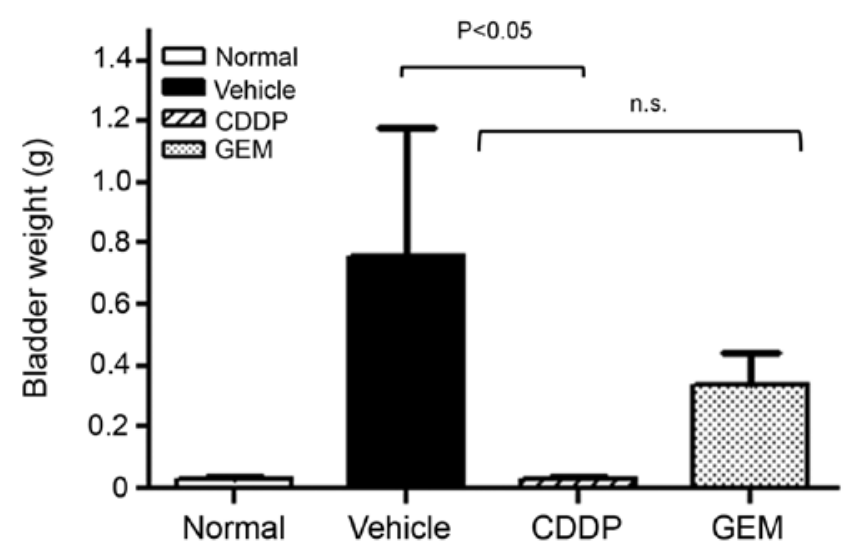

Figure 5. Bladder weights at day 21 after transplantation in the vehicle and chemotherapy groups. The CDDP-treated group $(n=8)$ demonstrated significantly lower bladder weight than the vehicle-treated group $(n=8)$. $\mathrm{P}<0.05$; Vehicle vs. CDDP; n.s., vehicle vs. GEM. CDDP, cisplatin; GEM, gemcitabine.

that progressed to superficial bladder carcinoma, CIS covering the urinary epithelium, and finally, to invasive carcinoma. Moreover, tumors were highly responsive to CDDP. This model will prove useful for the investigation of invasion and metastasis mechanisms as well as for the development of improved treatment strategies.
Recurrent bladder cancer after clinical treatment is highly prone to malignancy and metastasis, a characteristic shared by our mouse model. In a previously reported mouse orthotopic bladder cancer model, the urinary bladder wall was intentionally injured to enhance the propensity for cancer growth and direct invasion into the submucosal layer $(12,27)$. Conversely, we made several modifications to mitigate bladder injury, including relatively shallow catheter insertion, light urethral ligation, trypsin solution prewarming, and body temperature maintenance. Keeping the catheter tip approximately $1 \mathrm{~cm}$ from the urethral meatus prevented damage to the bladder and the ensuing hemorrhage. However, cancer cell invasion into the lamina propria and submucosal layer still occurred as in the natural progression of human bladder cancer. Thus, these improvements helped better replicate the pathological features of recurrent bladder cancer.

Our experiments demonstrated that infusion of UM-UC-3 human urinary bladder carcinoma cells following trypsin pretreatment results in microtumor attachment on the lamina propria and ensuing malignant changes. Therefore, our mouse orthotopic bladder cancer model may be useful for evaluating not only progression mechanisms and drug efficacy but also methods aimed at preventing microtumor initiation and early-stage development after primary clinical treatment.

Currently available single chemotherapeutics show limited efficacy for recurrent and invasive bladder cancer; however, CDDP may be among the most effective drugs $(28,29)$. Therapeutic efficacy of GEM for bladder cancer has also been reported, and combination therapy with CDDP and GEM improves overall survival rates and complete response in cancer patients $(30,31)$. In our mouse model, CDDP demonstrated higher antitumor activity than GEM. Therefore, this model may be useful for evaluating the therapeutic effects of drugs against recurrent and invasive bladder cancer.

We speculate that the strong efficacy of CDDP is due to two-way exposure through blood flow and urine because excretion via the kidney is the main pathway for CDDP removal. Conversely, GEM is first inactivated by cytidine deaminase to form 2',2'-difluorodeoxycytidine before excretion, which may decrease its efficacy against bladder cancer.

In conclusion, our improved mouse orthotopic bladder cancer model recapitulates the major events of microtumor development and metastasis as well as the anticancer drug responses of recurrent human bladder cancer. Thus, it may prove to be a valuable tool for revealing histopathological markers for diagnosis and prognosis. In addition, this model could aid in elucidating the therapeutic mechanisms of existing agents and in developing novel preventive and antitumor treatments for recurrent bladder cancer.

\section{Acknowledgements}

The authors would like to thank Mr Takeshi Yamaura and Ms. Hiroko Nemoto (FUJIFILM Corporation, Japan) for their assistance.

\section{Funding}

The present study was supported by FUJIFILM. 


\section{Availability of data and materials}

The datasets used and/or analyzed during the present study are available from the corresponding author on reasonable request.

\section{Authors' contributions}

TH, TN and YS established the mouse model and analyzed and performed the histological examination. CK confirmed the pathology data. TN and TH drafted the manuscript. YS supervised the study. All authors read and approved the final manuscript.

\section{Ethics approval and consent to participate}

All animal experiments were approved by the FUJIFILM Animal Experimentation Committee (experimental protocol no. I28L027NP; 2016/11/14).

\section{Patient consent for publication}

Not applicable.

\section{Competing interests}

The authors declare that they have no competing interests.

\section{References}

1. Lynch CF and Cohen MB: Urinary system. Cancer 75: 316-329, 1995

2. Barocas DA and Clark PE: Bladder cancer. Curr Opin Oncol 20: 307-314, 2008

3. Kirkali Z, Chan T, Manoharan M, Algaba F, Busch C, Cheng L, Kiemeney L, Kriegmair M, Montironi R, Murphy WM, et al: Bladder cancer: Epidemiology, staging and grading, and diagnosis. Urology 66 (Suppl): S4-S34, 2005.

4. Jacobs BL, Lee CT and Montie JE: Bladder cancer in 2010: How far have we come? CA Cancer J Clin 60: 244-272, 2010.

5. Brawn PN: The origin of invasive carcinoma of the bladder. Cancer 50: 515-519, 1982

6. Kanematsu A, Tsuji Y, Kanba H, Noguchi T, Kamoto T and Okabe T: The sensitivity and clinical implications of periodical bladder biopsy following transurethral resection of superficial bladder transitional cell carcinoma. Hinyokika kiyo. Acta urologica Japonica (Japanese) 47: 1-4, 2001.

7. Koss LG, Nakanishi I and Freed SZ: Nonpapillary carcinoma in situ and atypical hyperplasia in cancerous bladders: Further studies of surgically removed bladders by mapping. Urology 9: 442-455, 1977.

8. Koss LG, Tiamson EM and Robbins MA: Mapping cancerous and precancerous bladder changes. A study of the urothelium in ten surgically removed bladders. JAMA 227: 281-286, 1974.

9. Zincke H,Utz DC and Farrow GM: Review of mayo clinic experience with carcinoma in situ. Urology 26 (Suppl): S39-S46, 1985.

10. Kamat AM, Flaig TW, Grossman HB, Konety B, Lamm D, O'donnell MA, Uchio E, Efstathiou JA and Taylor III JA: Expert consensus document: Consensus statement on best practice management regarding the use of intravesical immunotherapy with BCG for bladder cancer. Nat Rev Urol 12: 225-235, 2015.

11. Gasión JP and Cruz JF. Improving efficacy of intravesical chemotherapy. Eur Urol 50: 225-234, 2006.

12. Huebner D, Rieger C, Bergmann R, Ullrich M, Meister S, Toma M, Wiedemuth R, Temme A, Novotny V, Wirth MP, et al: An orthotopic xenograft model for high-risk non-muscle invasive bladder cancer in mice: Influence of mouse strain, tumor cell count, dwell time and bladder pretreatment. BMC Cancer 17: 790, 2017.

13. Matsumoto R, Tsuda M, Wang L, Maishi N, Abe T, Kimura T, Tanino M, Nishihara H, Hida K, Ohba Y, et al: Adaptor protein CRK induces epithelial-mesenchymal transition and metastasis of bladder cancer cells through $\mathrm{HGF} / \mathrm{c}-\mathrm{Met}$ feedback loop. Cancer Sci 106: 709-717, 2015.
14. van der Horst G, van Asten JJ, Figdor A, van den Hoogen C, Cheung H, Bevers RF, Pelger RC and van der Pluijm G: Real-time cancer cell tracking by bioluminescence in a preclinical model of human bladder cancer growth and metastasis. Eur Urol 60: 337-343, 2011.

15. Nogawa M, Yuasa T, Kimura S, Tanaka M, Kuroda J, Sato K Yokota A, Segawa H, Toda Y, Kageyama S, et al: Intravesical administration of small interfering RNA targeting PLK-1 successfully prevents the growth of bladder cancer. J Clin Invest 115: 978-985, 2005.

16. Kim CJ, Tambe Y, Mukaisho KI, Sugihara H, Kageyama S, Kawauchi A and Inoue H: Periostin suppresses in vivo invasiveness via PDK1/Akt/mTOR signaling pathway in a mouse orthotopic model of bladder cancer. Oncol Lett 13: 4276-4284, 2017.

17. Chan E, Patel A, Heston W and Larchian W: Mouse orthotopic models for bladder cancer research. BJU Int 104: 1286-1291, 2009.

18. Seager C, Puzio-Kuter AM, Cordon-Cardo C, McKiernan J and Abate-Shen C: Mouse models of human bladder cancer as a tool for drug discovery. Curr Protoc Pharmacol 49: 14, 2010.

19. Zhang N, Li D, Shao J and Wang X: Animal models for bladder cancer: The model establishment and evaluation (Review). Oncol Lett 9: 1515-1519, 2015.

20. Kobayashi T, Owczarek TB, McKiernan JM and Abate-Shen C: Modelling bladder cancer in mice: Opportunities and challenges. Nat Rev Cancer 15: 42-54, 2015.

21. Shimada K, Nakamura M, Anai S, De Velasco M, Tanaka M, Tsujikawa K, Ouji Y and Konishi N: A novel human AlkB homologue, ALKBH8, contributes to human bladder cancer progression. Cancer Res 69: 3157-3164, 2009.

22. Zhou JH, Rosser CJ, Tanaka M, Yang M, Baranov E, Hoffman RM and Benedict WF: Visualizing superficial human bladder cancer cell growth in vivo by green fluorescent protein expression. Cancer Gene Ther 9: 681-686, 2002.

23. JoVE Science Education Database. Lab Animal Research. Anesthesia Induction and Maintenance. JoVE, Cambridge, MA, 2019.

24. Kameoka S, Matsumoto K, Kai Y, Yonehara Y, Arai Y and Honda K: Establishment of temporomandibular joint puncture technique in rats using in vivo micro-computed tomography (R_mCT(R)). Dentomaxillofac Radiol 39: 441-445, 2010.

25. Takahashi R, Yokobori T, Osone K, Tatsuki H, Takada T, Suto T, Yajima R, Kato T, Fujii T, Tsutsumi S, et al: Establishment of a novel method to evaluate peritoneal microdissemination and therapeutic effect using luciferase assay. Cancer Sci 107: 341-346, 2016.

26. Higuchi T, Yokobori T, Naito T, Kakinuma C, Hagiwara S, Nishiyama $\mathrm{M}$ and Asao T: Investigation into metastatic processes and the therapeutic effects of gemcitabine on human pancreatic cancer using an orthotopic SUIT2 pancreatic cancer mouse model. Oncol Lett 15: 3091-3099, 2017.

27. Dobek GL and Godbey WT: An orthotopic model of murine bladder cancer. J Vis Exp pii: 2535, 2011.

28. Sternberg CN, de Mulder PH, Schornagel JH, Theodore C, Fossa SD, Van Oosterom AT, Witjes F, Spina M, Van Groeningen CJ, De Balincourt C, et al: Randomized phase III trial of high-dose-intensity methotrexate, vinblastine, doxorubicin, and cisplatin (MVAC) chemotherapy and recombinant human granulocyte colony-stimulating factor versus classic MVAC in advanced urothelial tract tumors: European Organization for Research and Treatment of Cancer Protocol no. 30924. J Clin Oncol 19: 2638-2646, 2001.

29. GhataliaP,Zibelman M,Geynisman DM andPlimackE: Approved checkpoint inhibitors in bladder cancer: which drug should be used when? Ther Adv Med Oncol 10: 1758835918788310, 2018

30. von der Maase H, Hansen SW, Roberts JT, Dogliotti L, Oliver T, Moore MJ, Bodrogi I, Albers P, Knuth A, Lippert CM, et al: Gemcitabine and cisplatin versus methotrexate, vinblastine, doxorubicin, and cisplatin in advanced or metastatic bladder cancer: Results of a large, randomized, multinational, multicenter, phase III study. J Clin Oncol 18: 3068-3077, 2000.

31. von der Maase H, Sengelov L, Roberts JT, Ricci S, Dogliotti L, Oliver T, Moore MJ, Zimmermann A and Arning M: Long-term survival results of a randomized trial comparing gemcitabine plus cisplatin, with methotrexate, vinblastine, doxorubicin, plus cisplatin in patients with bladder cancer. J Clin Oncol 23: 4602-4608, 2005.

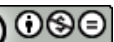

This work is licensed under a Creative Commons Attribution-NonCommercial-NoDerivatives 4.0 International (CC BY-NC-ND 4.0) License. 